Behavioral/Systems/Cognitive

\title{
Purkinje Cell Synapses Target Physiologically Unique Brainstem Neurons
}

\author{
Chris Sekirnjak, ${ }^{1}$ Bryce Vissel, ${ }^{2}$ Jacob Bollinger, ${ }^{1}$ Michael Faulstich, ${ }^{1}$ and Sascha du Lac ${ }^{1}$ \\ ${ }^{1}$ Systems Neurobiology Laboratories and ${ }^{2}$ Molecular Neurobiology Laboratories, The Salk Institute for Biological Studies, La Jolla, California 92037
}

The cerebellum controls motor learning via Purkinje cell synapses onto discrete populations of neurons in the deep cerebellar nuclei and brainstem vestibular nuclei. In the circuitry that subserves the vestibulo-ocular reflex, the postsynaptic targets of Purkinje cells, termed flocculus target neurons (FTNs), are thought to be a critical site of learning. Little is known, however, about the intrinsic cellular properties of FTNs, which are sparsely distributed in the medial vestibular nucleus. To identify these neurons, we used the L7 promoter to express a tau-green fluorescent protein fusion protein selectively in Purkinje cells. Fluorescent Purkinje cell axons and terminal boutons surrounded the somata and proximal dendrites of a small subset of neurons, presumed FTNs, in the medial vestibular nucleus. Targeted intracellular recordings revealed that FTNs fired spontaneously at high rates in brain slices (mean, $47 \mathrm{spikes} / \mathrm{sec}$ ) and exhibited dramatic postinhibitory rebound firing after the offset of membrane hyperpolarization. These intrinsic firing properties were exceptional among brainstem vestibular nucleus neurons but strikingly similar to neurons in the deep cerebellar nuclei, indicating a common role for intrinsic firing mechanisms in cerebellar control of diverse behaviors.

Key words: cerebellum; flocculus target neuron; vestibulo-ocular reflex; medial vestibular nucleus; postinhibitory rebound; motor learning; GFP

\section{Introduction}

The cerebellum is involved in a remarkably wide range of behaviors, from fine-tuning reflexive movements to acquiring new motor skills to cognition. The cerebellar cortex influences most behaviors via inhibitory Purkinje cell synapses onto neurons in the deep cerebellar nuclei (DCN). In the circuitry for the vestibuloocular reflex (VOR), Purkinje cells synapse onto a scattered population of neurons in the brainstem vestibular nuclei that have been implicated in motor learning (Lisberger and Pavelko, 1988; Lisberger et al., 1994b; Partsalis et al., 1995).

The VOR provides one of the best understood models of motor learning in vertebrates. Image stability on the retina during head movements requires that the VOR produce eye movements that compensate for motion of the head. Persistent image motion during head movements results in a simple form of motor learning that recalibrates the VOR via cerebellar-dependent mechanisms of plasticity (for review, see du Lac et al., 1995; Raymond et al., 1996). Recordings of neurons in the circuitry for the VOR have revealed physiological correlates of motor learning both in Purkinje cells of the cerebellar flocculus (Miles et al., 1980; Watanabe, 1984; Lisberger et al., 1994c) and in their postsynaptic partners, flocculus target neurons (FTNs) (Lisberger and Pavelko, 1988; Lisberger et al., 1994b; Partsalis et al., 1995). FTNs

Received Mar. 27, 2003; revised May 22, 2003; accepted May 23, 2003.

This work was supported by National Institutes of Health Grants EY11027 (to S.d.L.) and MH58880 (to Steve Heinemann) and by a fellowship from the Sloan/Swartz Center for Theoretical Neurobiology at the Salk Institute (to C.S.) We thank John Thomas for providing the tau-GFP construct, Setareh Moghadam for assistance, and Steve Heinemann for support. We also thank Ed Callaway, Rich Krauzlis, and Larry Squire for comments on this manuscript.

Correspondence should be addressed to Dr. Sascha du Lac, SNL-D, The Salk Institute for Biological Studies, 10010 North Torrey Pines Road, La Jolla, CA 92037. E-mail: sascha@salk.edu.

Copyright $\odot 2003$ Society for Neuroscience $\quad 0270-6474 / 03 / 236392-07 \$ 15.00 / 0$ are brainstem interneurons in the VOR circuit that are monosynaptically inhibited by floccular Purkinje cells; they receive excitatory drive from vestibular nerve afferents and convey this head motion information directly to ocular motoneurons (du Lac et al., 1995; Highstein, 1998). The dramatic changes in FTN firing responses to head movements observed after motor learning in the VOR have suggested that VOR learning results from activitydependent modification of synapses onto FTNs or from changes in FTN intrinsic excitability (du Lac et al., 1995).

Similarities between the cerebellar control of motor learning in the VOR and classical conditioning of the eyelid response have suggested that common algorithms and mechanisms may underlie adaptive regulation of many different behaviors (Raymond et al., 1996; Mauk, 1997). Neurons in the DCN (which control eyelid conditioning and most other behaviors) are exceptional among CNS neurons in their combination of high spontaneous firing rates in vitro and strong postinhibitory rebound firing (Jahnsen, 1986; Mouginot and Gahwiler, 1995; Aizenman and Linden, 1999; Raman et al., 2000). Rebound firing controls synaptic plasticity in DCN neurons (Aizenman et al., 1998) and plays an integral role in some models of cerebellar-dependent learning (Mauk, 1997; Mauk and Donegan, 1997), suggesting that the intrinsic membrane properties of both DCN neurons and FTNs may be specialized for cerebellar-dependent plasticity. Progress toward a cellular analysis of FTNs has been hampered, however, by the difficulty in identifying these neurons, which are sparsely distributed within the vestibular nuclei (Sato et al., 1988; Babalian and Vidal, 2000).

We describe a new approach to identifying FTNs for cellular physiological analyses. The L7 promoter (Oberdick et al., 1990) was used to drive Purkinje cell-specific expression of a tau-green 
fluorescent protein (GFP) fusion protein in transgenic mice. Within the vestibular nuclei, a small population of Purkinje cell recipient neurons could be distinguished by the dense plexus of fluorescent axons and terminals surrounding their somata and proximal dendrites. Targeted intracellular recordings in brain slices revealed that these neurons are physiologically unique among brainstem vestibular neurons but remarkably similar to DCN neurons, implying common cellular rules across diverse behaviors for cerebellar Purkinje cell control of movement and motor learning.

\section{Materials and Methods}

L7-tau-GFP mice. To generate mice with green fluorescent Purkinje cells, we made a transgene comprising the promoter of the L7 gene (Oberdick et al., 1990) fused to cDNA encoding the tau-GFP fusion protein. The tau-GFP cDNA, similar to the previously reported tau-lacZ cDNA (Callahan and Thomas, 1994), is a fusion of three cDNAs encoding the following proteins: (1) the bovine microtubule-binding protein tau; (2) six copies of a myc sequence; and (3) GFP. The transgene was generated by ligating the cDNA encoding tau-GFP into a unique BamH1 site engineered into the $\mathrm{L} 7$ gene for this purpose. EcoR1 restriction sites were used to isolate the L7-tau-GFP fusion DNA sequences from the bluescript vector. The injection of the L7-tau-GFP transgene into BALB/c mouse embryos was performed by the Salk Institute core facility. Southern blot hybridization analysis of tail DNA from these pups revealed that seven of 50 pups born contained the transgene integrated into their genomic DNA. These pups were used as founders of seven lines of L7-tau-GFP mice, four of which were used in the present study. All lines were backcrossed to C57/bl6 for one to three generations before physiological analyses.

Immunohistochemistry and cell counts. Mice were deeply anesthetized with Nembutal and perfused transcardially with $0.1 \mathrm{M}$ PBS, followed by $4 \%$ paraformaldehyde in PBS. Brains were post-fixed for 30-60 min and sunk in $30 \%$ sucrose overnight. Frozen coronal or sagittal sections were cut on a Microm sliding microtome at a thickness of 10-30 $\mu \mathrm{m}$. After repeated rinsing in PBS, the sections were blocked with $2 \%$ normal goat serum, $1 \% \mathrm{BSA}$, and $0.3 \%$ Triton $\mathrm{X}-100$ for $45 \mathrm{~min}$ to minimize nonspecific labeling. The tissue was incubated in mouse anti-NeuN monoclonal antibodies (Chemicon, Temecula, CA) at 1:200 in working buffer $(0.1 \times$ strength blocking buffer) overnight at $4^{\circ} \mathrm{C}$. After several washes, secondary antibodies (Cy3-conjugated goat anti-mouse, 1:100; Chemicon) were applied in working buffer for $60 \mathrm{~min}$ at room temperature. After additional washes in PBS, the sections were floated on glass slides, treated with an antifade gel containing 2.5\% DABCO (Sigma, St. Louis, MO), and coverslipped. Fluorescent images were obtained using a Hamamatsu CCD camera attached to an Olympus BX60 light microscope with a $4 \times$ [numerical aperture (NA), 0.13 ], $10 \times(\mathrm{NA}, 0.3), 40 \times(\mathrm{NA}, 1.00)$, or $100 \times(\mathrm{NA}, 1.35)$ objective lens.

Estimates of the number of medial vestibular nucleus (MVN) neurons surrounded by GFP-positive terminal outlines were obtained from fluorescent Nissl-stained tissue (NeuroTrace; Molecular Probes, Eugene, OR) sectioned at $30 \mu \mathrm{m}$. To distinguish neurons from glial cell bodies, sections were counterstained with the nuclear marker $4^{\prime}, 6^{\prime}$-diamidino2-phenylindole (DAPI; Sigma). Nissl-positive structures, labeled in red, were identified as neurons if proximal processes could be discerned. Small neurons with no identifiable processes were distinguished from glial cells using the following criteria (Williams and Rakic, 1988): neurons contained abundant Nissl substance, neuronal nuclei (labeled in blue with DAPI) were round or oval, and neuronal nucleoli were large and prominent. Counts of the total number of neurons per unilateral section were performed in three representative sections of the rostral MVN that were subdivided into multiple rectangular counting frames $(150 \times 125 \mu \mathrm{m})$. Neurons were counted only if their nuclei were completely within the margins of the counting frame. Green fluorescent Purkinje cell terminal outlines were identified by focusing through the tissue while searching for a dense plexus of fluorescent, bouton-like structures (Figs. $1 E, 2 C$ ). Terminal outlines were counted both in Nissl-stained and NeuN-stained sections.
Flocculectomies. Surgical ablation of the floccular lobe (including the flocculus and paraflocculus) was performed under isofluorane anesthesia. The temporal bone was exposed rostral to the posterior canal and dorsal to the horizontal canal, and the bony plate overlying the paraflocculus was removed. The floccular lobe was aspirated unilaterally with a blunted 23-gauge needle, and the cavity was sealed with gel foam. After $20-30 \mathrm{~d}$, the brains were removed and sectioned at $50 \mu \mathrm{m}$. In two lesioned mice in which complete removal of the flocculus could be verified, the number of terminal outlines in the MVN ipsilateral and contralateral to the lesion were counted.

Electrophysiology. Slices were prepared from mice, 16-19 d old, as described previously (Sekirnjak and du Lac, 2002). Whole-cell patch recordings were made in a submersion chamber perfused with carbogenated artificial SCF (ACSF) warmed to $31-33^{\circ} \mathrm{C}$. Neurons were visualized at $40-80 \times$ with an infrared differential interference contrast microscope (Olympus) at depths of $10-50 \mu \mathrm{m}$ below the slice surface. GFP signal was detected using a xenon light source and a Sensicam CCD camera. Continuous fluorescence images were displayed using SlideBook software (Intelligent Imaging Innovations, Denver, CO). Recordings were made with an AxoClamp 2B amplifier (Axon Instruments, Foster City, CA) in current-clamp mode. Access resistance was checked and compensated regularly throughout each experiment. Signals were filtered at $10 \mathrm{kHz}$, digitized at $20 \mathrm{kHz}$, and recorded using a Macintosh G4 computer. A liquid junction potential calculated at $14 \mathrm{mV}$ (using Jpcalc) was subtracted from all membrane potentials. Spontaneous firing rates were determined from stable firing epochs of at least $5 \mathrm{sec}$. Action potential parameters were obtained from averages of at least 50 spikes obtained when neurons were maintained to fire at $10-15 \mathrm{~Hz}$ with DC injection; bridge balance was checked and adjusted under all conditions of intracellular current injection. Firing response gains were calculated as the slope of the relationship between evoked firing and input current applied for $1 \mathrm{sec}$. Postinhibitory rebound firing was obtained after $1 \mathrm{sec}$ steps of current that hyperpolarized the membrane to a mean of $30 \mathrm{mV}$ below the resting potential averaged when neurons fired at 10 spikes/sec; this protocol elicits maximal rebound firing and was used to compare neurons in this study with those reported previously (Sekirnjak and du Lac, 2002). For dye filling of cells, tetramethylrhodamine dextran (Molecular Probes), $0.1 \mathrm{mg} / \mathrm{ml}$, was included in the internal recording solution. Soma sizes were estimated from images of dye-filled neurons acquired during whole-cell recordings; cell bodies were approximated by an ellipse, and the elliptical area was calculated. Statistical analyses of physiological and anatomical parameters of neuronal populations were performed using the Mann-Whitney $U$ test. Population data are reported as means \pm SEs.

Solutions. ACSF contained $124 \mathrm{~mm} \mathrm{NaCl}, 5 \mathrm{~mm} \mathrm{KCl}, 1.3 \mathrm{~mm} \mathrm{MgSO}_{4} 26$ mm NaHCO3, $2.5 \mathrm{~mm} \mathrm{CaCl} 2,1 \mathrm{~mm} \mathrm{NaH}{ }_{2} \mathrm{PO}_{4}$, and $11 \mathrm{~mm}$ dextrose. The glutamate receptor blocker kynurenic acid $(2 \mathrm{~mm})$ was added to the bath solution in all experiments. Aerated ACSF had a final $\mathrm{pH}$ of 7.4 and an osmolarity of $300 \mathrm{mOsm}$. The internal recording solution contained 140 mM K-gluconate, $8 \mathrm{~mm} \mathrm{NaCl}, 10 \mathrm{~mm}$ HEPES, $0.1 \mathrm{~mm}$ EGTA, 2 mм Mg-ATP, and 0.3 mm Na-GTP. The $\mathrm{pH}$ was adjusted to 7.2-7.5, and the osmolarity to $280-285 \mathrm{mOsm}$.

\section{Results}

We used the L7 promoter (Oberdick et al., 1990) to drive expression of a tau-GFP fusion protein in cerebellar Purkinje cells. The resulting GFP expression within the brain was restricted to the cerebellar cortex, deep cerebellar nuclei, and vestibular nuclei (Fig. 1A,B). Purkinje cell somata, dendrites, and axons throughout the cerebellum were strongly fluorescent (Fig. 1D), although the signal intensity could vary across cells within any given cerebellar lobule (Fig. 1C).

The majority of cerebellar Purkinje cells synapse onto neurons in the DCN; $90 \%$ of DCN neurons are contacted by Purkinje cell terminals, with an estimated convergence ratio of 26:1 (Palkovits et al., 1977). Consistent with the known anatomy, DCN neurons in L7-tau-GFP mice were completely enmeshed in fluorescent Purkinje cell axons and terminals (Fig. $1 F$ ). 

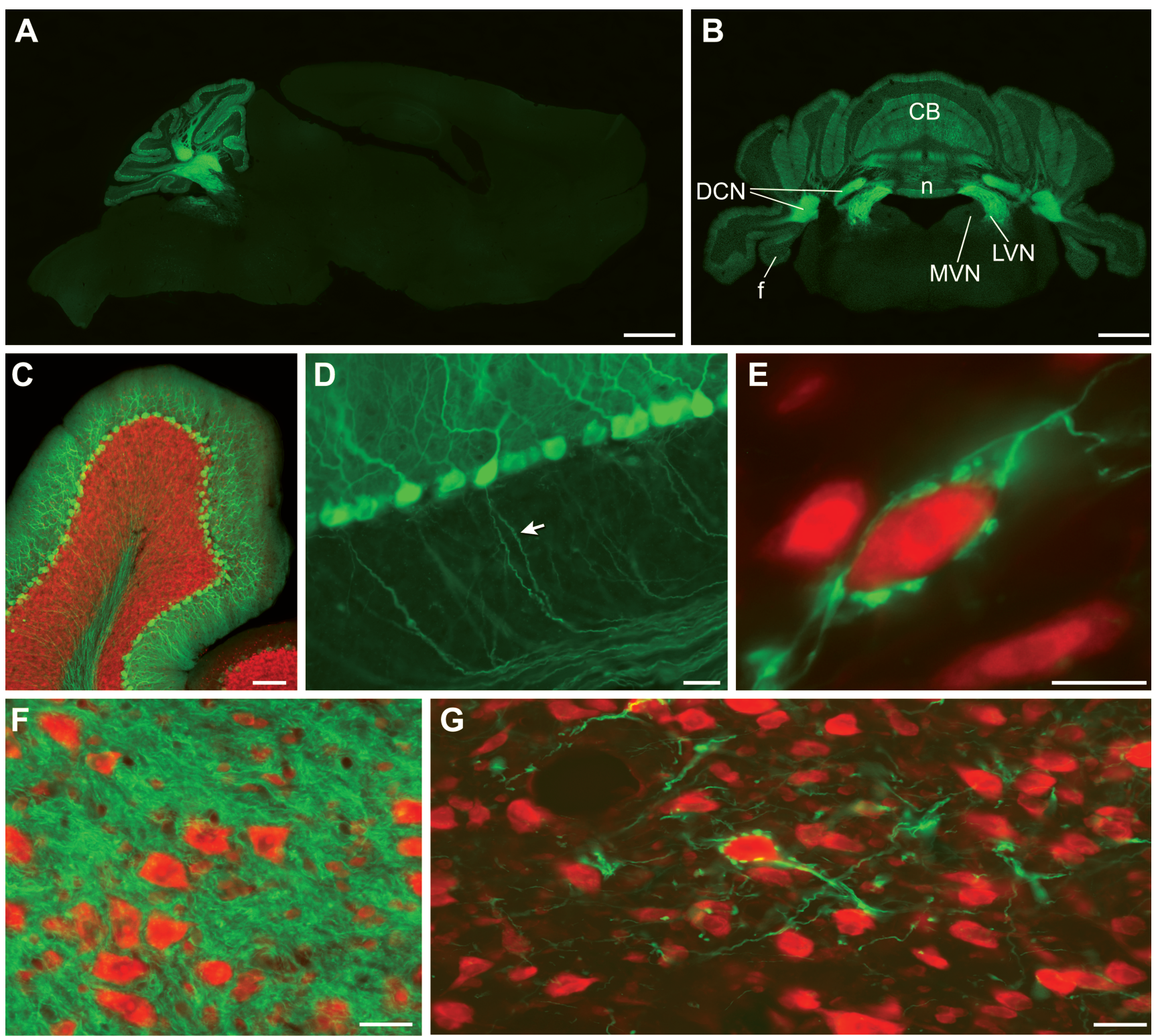

Figure 1. GFP expression in L7-tau-GFP mice. Sagittal $(A)$ and coronal $(B)$ sections indicate that GFP expression is restricted to the cerebellar cortex (CB), DCN, and vestibular nuclei in the brainstem [MVN, lateral vestibular nucleus (LVN), floccular lobe (f), and nodulus (n)]. C, A single cerebellar lobule showing GFP expression (green) in Purkinje cells. Antibody staining for the neuronal marker NeuN is shown in red. D, GFP expression is visible in Purkinje cell somata, dendrites, and axons (arrow). E, Green fluorescent terminals outline a red fluorescent NeuN-stained neuron in the MVN. F, Section through the lateral cerebellar nucleus, in which green fluorescent Purkinje cell axons and terminal surround red Nissl-stained neurons. G, Section through the MVN, showing relatively sparse green fluorescent axons converging on individual neurons, Nissl-stained red. Scale bars: $A, B, 1 \mathrm{~mm} ; C, 100 \mu \mathrm{m} ; D, F, G, 25 \mu \mathrm{m} ; E, 10 \mu \mathrm{m}$.

In contrast, GFP-positive axons and terminals were relatively sparse in the MVN (Fig. $1 \mathrm{gm}$ ), which contains neurons that mediate cerebellar-dependent learning in the VOR. Purkinje cell axons and terminals formed occasional basket-like structures within the MVN that resembled the outlines of neurons (Fig. $1 E, G)$. GFP-positive terminal outlines were formed by multiple Purkinje cell axons converging on an individual neuron. Purkinje cell synaptic boutons were $1-4 \mu \mathrm{m}$ in diameter, resembled beads on a string, and contacted the somata and proximal dendrites of target neurons (Fig. 1E). Double labeling with antibodies against the neuronal marker NeuN revealed that these basket-like structures surrounded the somata and proximal dendrites of a small number of MVN neurons (Fig. $1 E$ ). Neurons surrounded by such fluorescent Purkinje cell terminal outlines were concentrated in the rostral portion of the MVN and comprised $\sim 1 \%$ of all MVN neurons: an average of $3.5 \pm 0.6$ neurons per section were sur- rounded by terminal outlines in sections containing an average of 376 Nissl-stained neurons ( $n=42$ sections in seven animals; see "Materials and Methods").

Two distinct portions of the cerebellum project to the vestibular nuclei, the floccular lobe and the nodulus. Given the critical role of the flocculus and FTNs in VOR plasticity (Ito, 1982; Lisberger and Pavelko, 1988; Highstein, 1998), we focused on identifying FTNs. To determine the locations of FTNs, we made unilateral lesions of the floccular lobe (Fig. 2A) and compared the resulting pattern of Purkinje cell terminals in the ipsilateral and contralateral MVNs (Purkinje cells project exclusively ipsilaterally). Although GFP-positive terminals remained near the border of the fourth ventricle, they were almost completely absent in the ventrolateral portion of the vestibular nucleus ipsilateral to the lesion (Fig. 2D). Within the region of the rostral MVN outlined in Figure 2B, the side to the flocculectomy had 95\% fewer termi- 

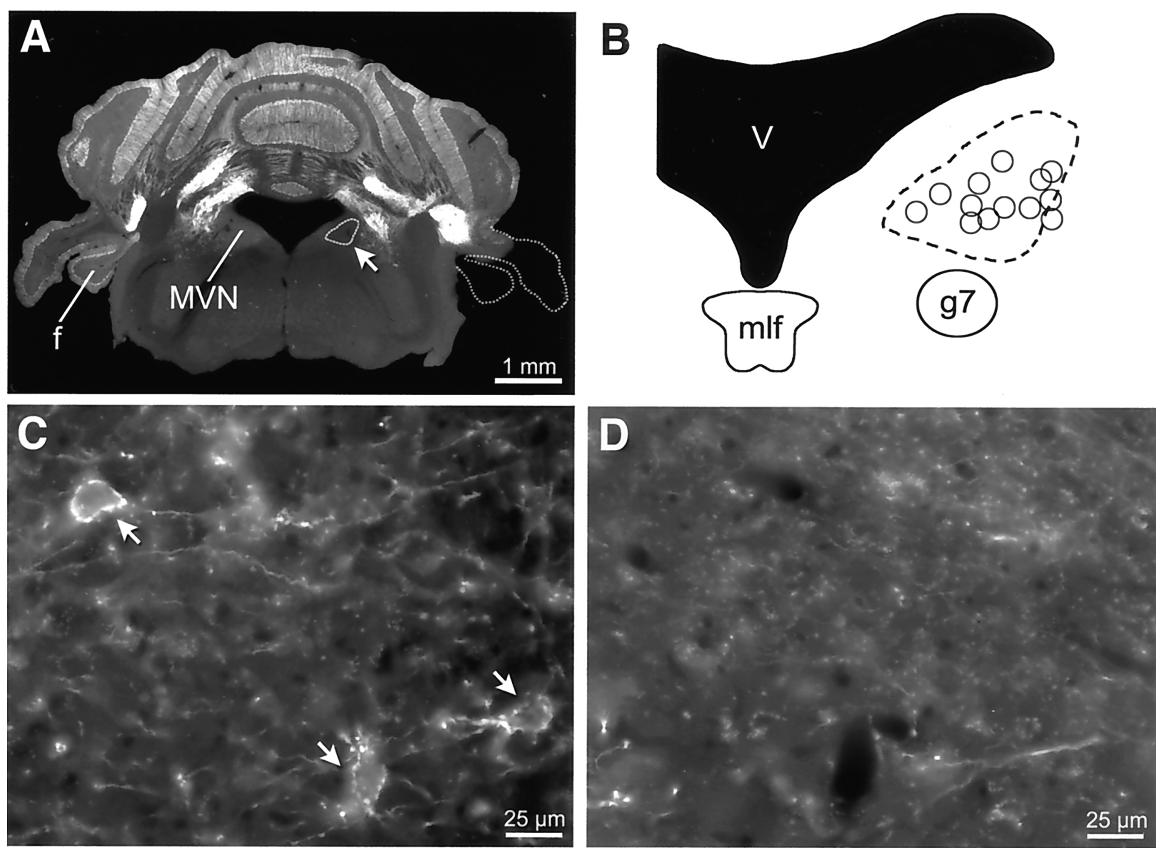

Figure 2. Distribution of Purkinje cell terminal outlines after unilateral flocculectomy. A, GFP signal in a coronal section of an adult mouse in which the right flocculus ( $f$ ) and paraflocculus were surgically ablated. The dashed line on the right outlines the portion of the cerebellum that was ablated. The region of the MVN encircled on the right side (arrow) corresponds with the dashed region in $B$. $B$, Schematic of the MVN. The dashed line encircles the region in which terminal outlines were missing on the side ipsilateral to the lesion, as exemplified in $D$. The circles indicate the approximate locations of intracellularly recorded FTNs described in this study. $C$, High-magnification view of the center of the region outlined in $B$, taken on the side contralateral to the floccular lesion. Three FTNs are evident (arrows). D, The same location as in C, but on the side ipsilateral to the lesion, is devoid of terminal outlines. Scale bars: $A, 1 \mathrm{~mm} ; C, D, 25 \mu \mathrm{m}$.

nal outlines than did the contralateral side (248 outlines contralateral vs 12 ipsilateral; $n=2$ animals). This finding indicates that the Purkinje cell terminals normally found in this region are predominantly floccular in origin, making their postsynaptic targets FTNs.

To confirm that the MVN neurons surrounded by fluorescent terminals were, in fact, inhibited by Purkinje cells, we prepared brain slices and used a combination of fluorescence and standard differential interference contrast microscopy to target presumed FTNs for electrophysiological recordings. An example of such a recording is shown in Figure 3. The neuron was filled intracellularly with rhodamine dextran. A glass-stimulating pipette, placed on a fluorescent axon coursing toward the recorded neuron, was used to evoke synaptic responses. Stimulation evoked IPSPs with short latencies $(<0.8 \mathrm{msec})$. Stimulating with the electrode moved 5-10 $\mu \mathrm{m}$ away from the fluorescent axon failed to evoke an IPSP, indicating that evoked IPSPs were mediated by Purkinje cells rather than by unlabeled axons. Similar results were obtained in each of four other FTNs tested.

To examine whether cerebellar target neurons in the MVN were physiologically distinct from other vestibular nucleus neurons, we made pairs of recordings from neurons surrounded by fluorescent terminals and from nearby neurons (within $200 \mu \mathrm{m}$ ) that were completely devoid of terminals around their somata (Fig. 4). Our recordings were restricted to the ventrolateral portion of the MVN (Fig. 2 B). We will refer to neurons with somatic Purkinje cell inputs as FTNs and those devoid of somatic inputs as non-FTNs, although it is possible that our presumed nonFTNs receive Purkinje cell inputs on distal dendrites. FTNs could not be distinguished from non-FTNs either on the basis of soma size (FTN soma area: $259 \pm 21 \mu \mathrm{m}^{2}, n=15$; non-FTN: $277 \pm 26$ $\left.\mu \mathrm{m}^{2}, n=16 ; p=0.81\right)$ or input resistance (FTN: $122 \pm 15 \mathrm{~m} \Omega, n=15$; non-FTN: $153 \pm 28 \mathrm{~m} \Omega, n=16 ; p=0.62$ ). However, the intrinsic firing properties of FTNs were strikingly different from those of non-FTNs.

FTNs had exceptionally high spontaneous firing rates compared with non-FTNs, as exemplified by the neurons in Figure $5 A$. The high firing rates of FTNs were not an artifact of dialysis during whole-cell patch recording: firing rates measured during cell-attached extracellular recordings and intracellular recordings were similar (43 \pm 11 and $47 \pm 7 ; n=8$ and 16 , respectively). Spontaneous firing rates measured intracellularly in FTNs were significantly higher than those of non-FTNs ( $24 \pm 8$ spikes/sec; $n=8 ; p<0.05$ ), half of which ( 8 of 16) did not fire spontaneously. Consistent with these differences in spontaneous rates, action potential thresholds were significantly lower in FTNs than in non-FTNs $(-53 \pm 1.0 \mathrm{mV}$ vs $-46.4 \pm 1.2$ $\mathrm{mV} ; p=0.001)$. As shown in Figure $5 B, C$, the afterhyperpolarization was also signficantly smaller in FTNs $(15.6 \pm .7 \mathrm{mV} ; n=$ 15) than in either neighboring non-FTNs $(20.5 \pm .9 \mathrm{mV} ; n=16 ; p<0.001)$ or a sample of unidentified MVN neurons (23.2 $\pm .5 \mathrm{mV}, n=146, p<0.0001)$. The high intrinsic spontaneous firing rates observed in FTNs may underlie the ability of these neurons to fire at rates of over $100 \mathrm{spikes} / \mathrm{sec}$ in the awake behaving animal (Lisberger et al., 1994a; Zhang et al., 1995) despite receiving tonic inhibitory drive from Purkinje cells that themselves fire spontaneously at equivalently high rates (Lisberger et al., 1994c).

Neurons in the circuit mediating the VOR receive excitatory synaptic inputs from vestibular nerve afferents, which respond to head movements by modulating their firing rates over an excep-

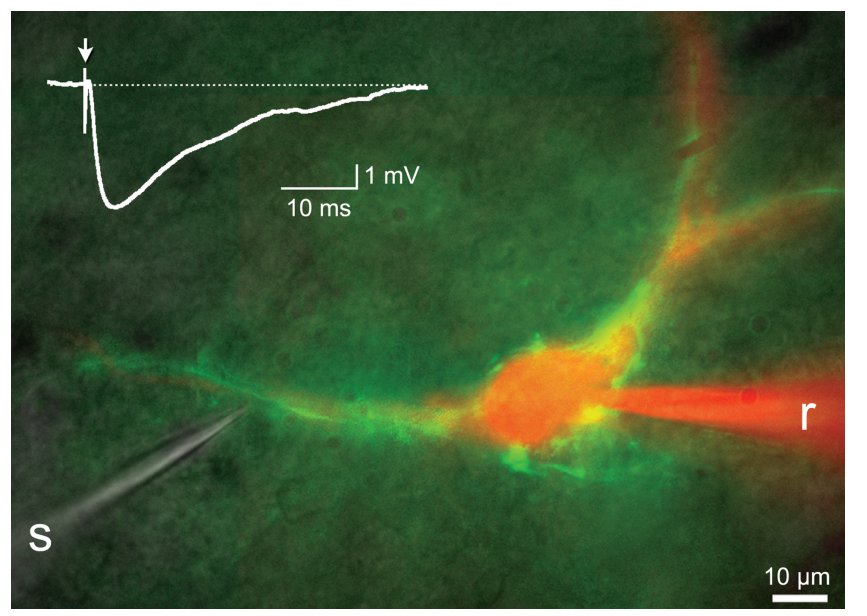

Figure 3. Purkinje cell synaptic inhibition in an FTN. Purkinje cell terminal clustering (green) identifies an FTN, labeled intracellularly with rhodamine dextran (red). The recording electrode ( $r$ ) can be seen on the right, and a stimulating electrode $(s)$ is on the left. The inset shows the average of 10 inhibitory postsynaptic potentials evoked by an $11 \mathrm{~V}$ stimulus. The dashed line indicates $-58 \mathrm{mV}$. Excitatory synaptic transmission was blocked by kynurenic acid ( $2 \mathrm{~mm}$ ). 

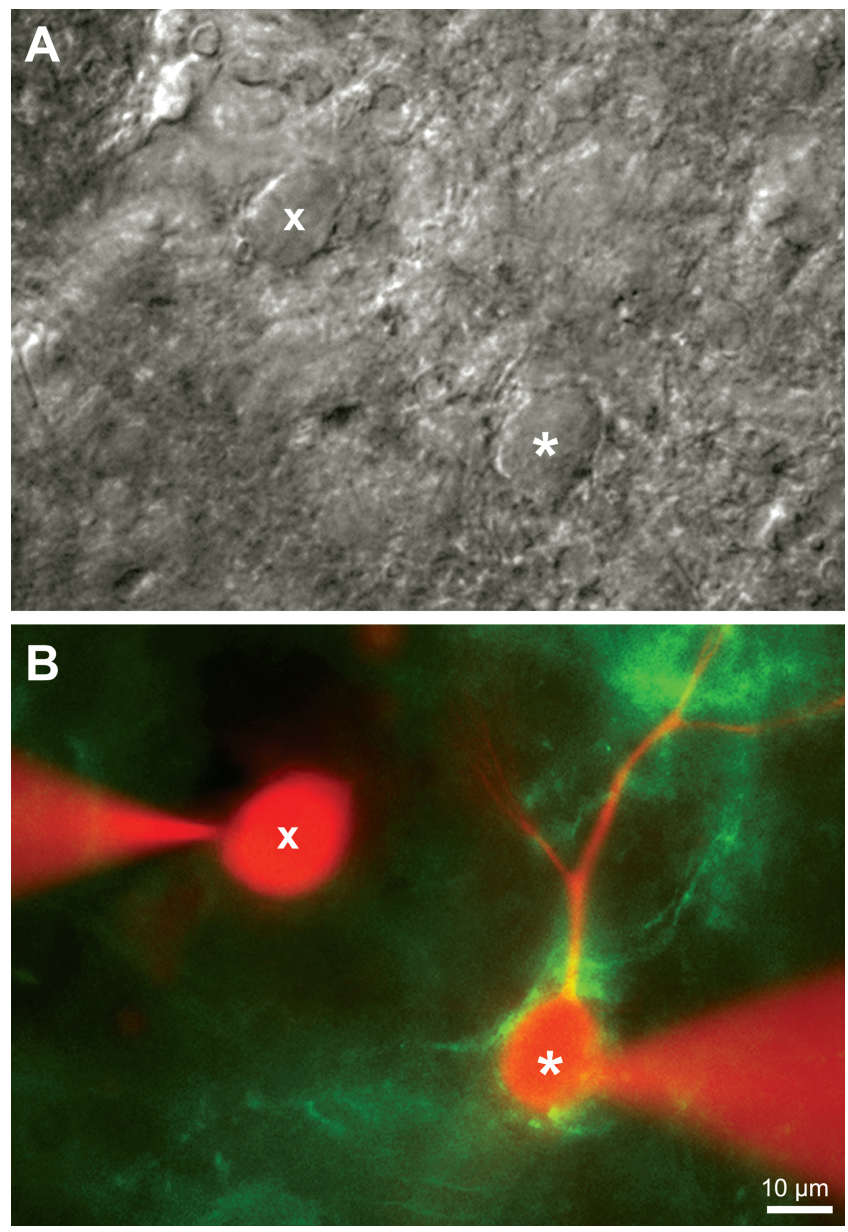

Figure 4. Matched intracellular recordings from FTNs and neighboring neurons. Differential interference contrast images $(A)$ and fluorescent images $(B)$ of an FTN $\left(^{*}\right)$ identified by GFP terminal clustering and a neighboring neuron $(\mathrm{x})$ that was not surrounded by GFP-positive terminals and was, therefore, a presumed non-FTN. Neurons were recorded sequentially and each labeled intracellularly with rhodamine dextran.

tionally wide range, from 0 to over 200 spikes/sec (Fernandez and Goldberg, 1971). We assessed the ability of FTNs to transmit depolarizing signals faithfully by examining their responses to depolarizing current injection. Figure 6 demonstrates that both FTNs and non-FTNs exhibited relatively little spike frequency adaptation, indicating that they process depolarizing somatic inputs with modest temporal filtering. FTNs and non-FTNs each fired linearly as a function of input current strength $\left(R^{2}=0.98 \pm\right.$ 0.005 and $0.98 \pm 0.004 ; n=11$ and 15 for FTNs and non-FTNs, respectively). As shown in Figure $6 B$, response gains (slope of the firing rate-input curve) were higher in FTNs (281 \pm 39 spikes/ $\mathrm{sec} / \mathrm{nA})$ than in non-FTNs $(138 \pm 14 \mathrm{spikes} / \mathrm{sec} / \mathrm{nA} ; p=0.0005)$, as were the maximal firing rates that could be sustained over $1 \mathrm{sec}$ of depolarization (FTNs, $236 \pm 15$ spikes/sec; non-FTNs, $173 \pm$ 19 spikes/sec; $p<0.01)$. These results indicate that FTNs respond sensitively and linearly over a wide range of depolarizing inputs, making them well suited to producing fine-tuned modulations of the VOR.

Purkinje cells provide powerful inhibitory synaptic drive to FTNs; in intact animals, FTNs firing as fast as $100 \mathrm{~Hz}$ are completely silenced after electrical stimulation of the flocculus with a single shock (du Lac and Lisberger, 1992; Zhang et al., 1995). We used hyperpolarizing current injection to investigate whether FTNs respond differently to hyperpolarizing inputs than do non-
A
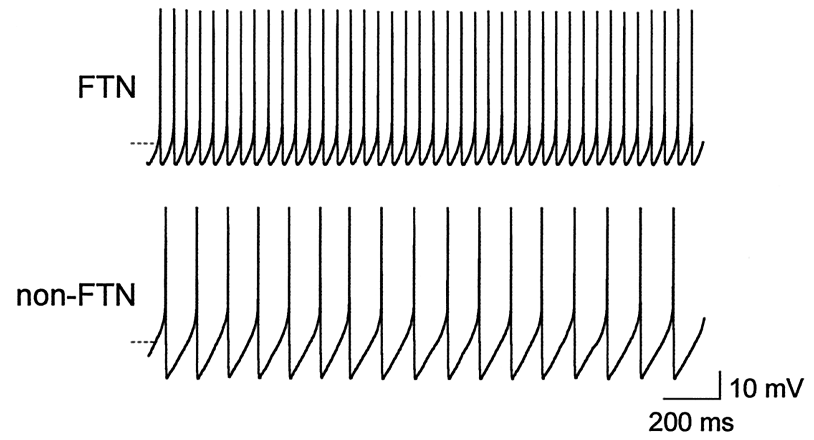

B

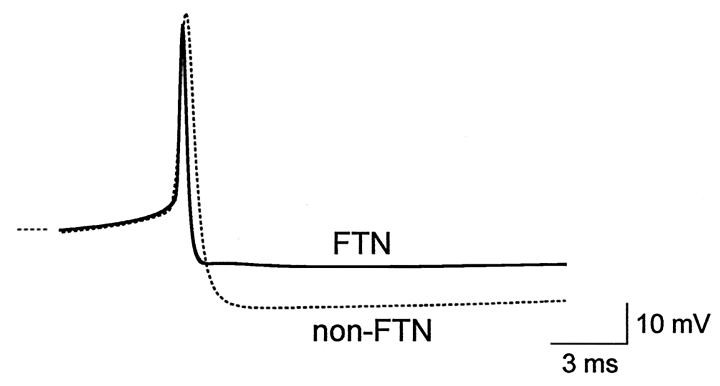

C

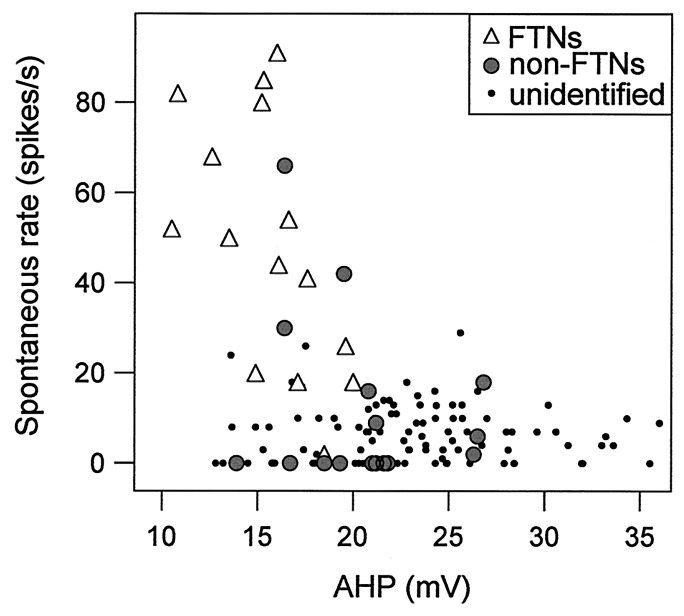

Figure 5. Fast intrinsic spontaneous firing in FTNs versus non-FTNs. $A$, Spontaneous action potentials in an FTN firing at 41 spikes/sec and a neighboring non-FTN firing at 18 spikes/sec. The dashed lines indicate $-60 \mathrm{mV}$. Synaptic transmission was blocked with kynurenic acid (2 mм). $B$, Action potentials from the same neurons as in $A$ are shown averaged from 5 sec epochs in which neurons were maintained at 12 spikes/sec with DC injection. The dashed line indicates $-60 \mathrm{mV}$. C, Spontaneous firing rate is plotted versus afterhyperpolarization amplitude in 15 FTNs (filled circles), 14 non-FTNs (open triangles), and 107 unidentified MVN neurons (dots) from a previous study (Sekirnjak and du Lac, 2002).

FTNs. After the offset of membrane hyperpolarization, FTNs displayed dramatic postinhibitory rebound firing, which peaked at 277 spikes/sec in the example shown in Figure $7 A$. In contrast, non-FTNs exhibited little or no rebound firing (Fig. 7A,B). Peak rebound firing across our sample of FTNs was $186 \pm 14$ spikes/ $\sec (n=16)$, significantly higher than that of either non-FTNs (19 \pm 7 spikes/sec; $n=16 ; p<0.0001)$, or of unidentified MVN neurons examined in a previous study $(19 \pm 2$ spikes/sec; $n=$ 108; $p<0.0001$; Sekirnjak and du Lac, 2002). Figure 7C plots peak postinhibitory rebound firing versus firing response gain for our sample of FTNs, neighboring non-FTNs, and unidentified 

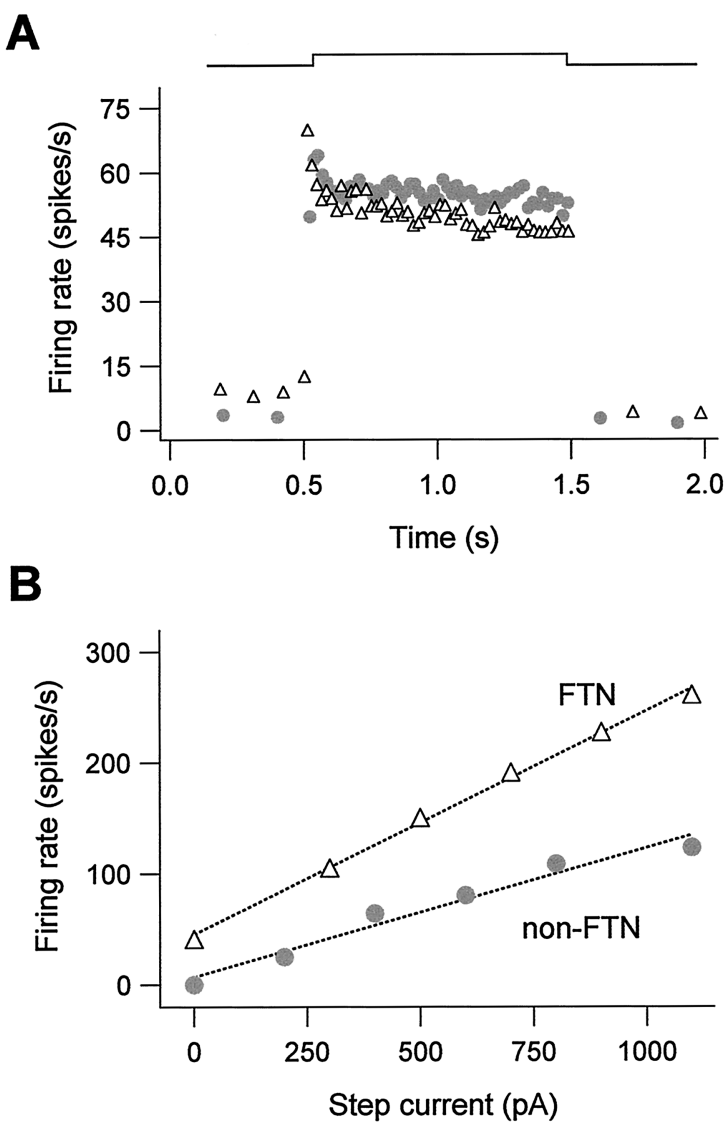

Figure 6. Wide and linear dynamic firing range in FTNs. A, Responses to intracellular depolarization with steps of current, plotted as instantaneous firing rate versus time, are shown for an FTN (triangles) and a neighboring non-FTN (circles). Neither neuron exhibited pronounced spike frequency adaptation. Current amplitudes were 100 pA (FTN) and 200 pA (non-FTN). B, Mean firing rate versus input current plotted for an FTN and a non-FTN, revealing linear firing responses in both cell types. The FTN could sustain firing rates of up to 261 spikes/sec.

MVN neurons, and shows that FTNs can be unambiguously distinguished from other MVN neurons by a combination of their high firing response gains and exceptionally strong postinhibitory rebound firing.

\section{Discussion}

We have identified an anatomically and physiologically unique population of brainstem neurons that mediate cerebellar control of the VOR. Purkinje cells in the floccular lobe of the cerebellum target synapses to the somata of a sparse population of MVN neurons. These FTNs have intrinsic firing properties that are exceptional among brainstem vestibular neurons but similar to Purkinje cell recipient neurons in the DCN. Our results indicate that cerebellar systems that subserve different behaviors use common cellular mechanisms and suggest that postinhibitory rebound firing mechanisms may play an integral role in cerebellar function.

The existence of the Purkinje cell-specific L7 promoter (Oberdick et al., 1990), coupled with the somatic distribution of synaptic boutons (De Zeeuw and Berrebi, 1995), enabled us to use presynaptic GFP expression to identify the postsynaptic targets of cerebellar Purkinje cells. Similar strategies could be used to identify the postsynaptic partners of other presynaptic neurons that target synapses to cell bodies. Our identification of FTNs was restricted to neurons with multiple labeled somatic boutons; we therefore may have underestimated the fraction of MVN neurons

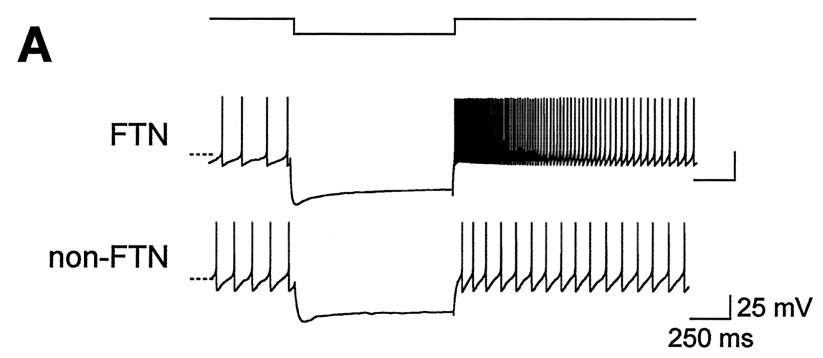

B
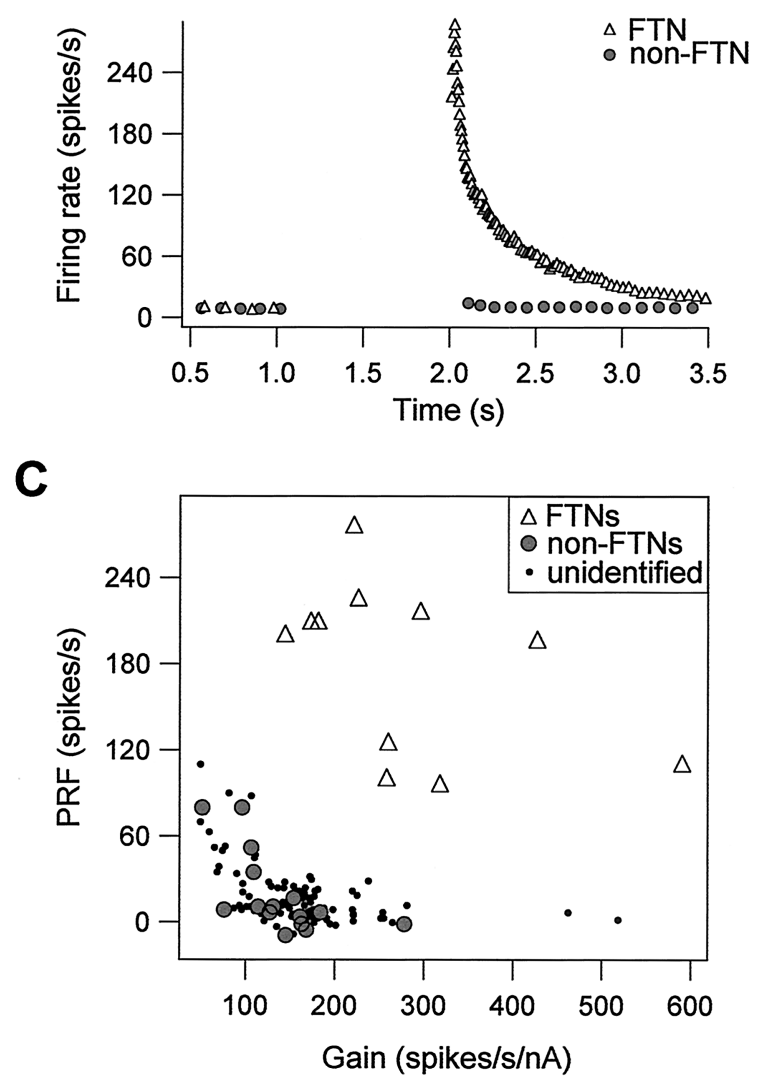

Figure 7. FTNs exhibit exceptionally strong postinhibitory rebound firing. $A$, Responses to intracellular hyperpolarization of an FTN and a neighboring non-FTN. The top trace shows time course of current injection (amplitude, $-500 \mathrm{nA}$ and $-200 \mathrm{nA}$ for the FTN and non-FTN, respectively). The bottom traces show membrane potential versus time; the dashed lines indicate $-60 \mathrm{mV}$. B, Instantaneous firing rate versus time for the examples shown. Resting firing was maintained at a standardized value of $\sim 10$ spikes/sec with DC injection. After the offset of membrane hyperpolarization, the FTN fired rebound action potentials that peaked at 277 spikes/sec, whereas the non-FTN exhibited little rebound firing (peak, 4 spikes/sec). C, Postinhibitory rebound firing (PRF) is plotted against firing response gain of 11 FTNs (open triangles), 15 non-FTNs (filled circles), and 108 unidentified MVN neurons (dots).

receiving floccular synapses. Studies in intact animals have reported that between 8 and $12 \%$ of MVN neurons recorded with extracellular electrodes were inhibited by stimulation of the flocculus (and therefore FTNs) (Sato et al., 1988), suggesting that a remarkably small population of neurons is responsible for cerebellar control of the VOR. Our data indicate that floccular Purkinje cells target densely clustered synapses to only $1 \%$ of the total population of MVN neurons.

The intrinsic firing properties of FTNs are well suited for mediating inhibitory cerebellar control of the VOR. Like other vestibular nucleus neurons, FTNs respond to excitatory drive with 
remarkably linear increases in firing rate to over 200 spikes/sec, enabling them to signal precise information about a wide range of head movement amplitudes and frequencies. FTNs are unique, however, both in their high spontaneous firing rates and in their exceptionally strong postinhibitory rebound firing. High intrinsic spontaneous rates, coupled with tonic depression of Purkinje cell synapses (Telgkamp and Raman, 2002), could account for the ability of FTNs to maintain a high tonic firing level in vivo at rates of 50-150 spikes/sec, despite receiving convergent and powerful inhibitory drive from Purkinje cells that themselves fire spontaneously at $50-150 \mathrm{~Hz}$. The dramatic postinhibitory rebound firing in FTNs reflects the activity of ionic currents that are activated by hyperpolarizing stimuli such as would occur under conditions of high firing rates in floccular Purkinje cells.

The similarities between intrinsic excitability of FTNs and of neurons in the DCN have implications for cerebellar-dependent motor learning. Like their counterparts in the vestibular nuclei, neurons in the DCN are studded with densely packed Purkinje cell synapses (Chan-Palay, 1977), fire spontaneously at high rates in vitro (Mouginot and Gahwiler, 1995; Aizenman et al., 1998; Raman et al., 2000), and exhibit pronounced postinhibitory rebound firing (Jahnsen, 1986; Aizenman et al., 1998; Aizenman and Linden, 1999). The finding that these anatomically distinct populations of neurons share a common electrophysiological signature implies that their membrane properties are specialized for transforming Purkinje cell inhibitory synaptic drive into the changes in postsynaptic signaling that give rise to motor learning. Postinhibitory rebound firing provides a mechanism for coupling Purkinje cell activity with increases in postsynaptic calcium that trigger synaptic plasticity in the DCN (Aizenman et al., 1998) and as such plays an integral role in some models of cerebellardependent learning (Mauk, 1997; Mauk and Donegan, 1997). Learning rules for VOR plasticity (Raymond and Lisberger, 1998) are similarly likely to depend on Purkinje cell control of intracellular calcium levels in FTNs. The L7-tau-GFP mice will enable future studies to test this hypothesis directly.

\section{References}

Aizenman CD, Linden DJ (1999) Regulation of the rebound depolarization and spontaneous firing patterns of deep nuclear neurons in slices of rat cerebellum. J Neurophysiol 82:1697-1709.

Aizenman CD, Manis PB, Linden DJ (1998) Polarity of long-term synaptic gain change is related to postsynaptic spike firing at a cerebellar inhibitory synapse. Neuron 21:827-835.

Babalian AL, Vidal PP (2000) Floccular modulation of vestibuloocular pathways and cerebellum-related plasticity: an in vitro whole brain study. J Neurophysiol 84:2514-2528.

Callahan CA, Thomas JB (1994) Tau- $\beta$-galactosidase, an axon-targeted fusion protein. Proc Natl Acad Sci USA 91:5972-5976.

Chan-Palay V (1977) Cerebellar dentate nucleus. Berlin, Heidelberg, New York: Springer.

De Zeeuw CI, Berrebi AS (1995) Postsynaptic targets of Purkinje cell terminals in the cerebellar and vestibular nuclei of the rat. Eur J Neurosci 7:2322-2333.

du Lac S, Lisberger SG (1992) Eye movements and brainstem neuronal responses evoked by cerebellar and vestibular stimulation in chicks. J Comp Physiol A 171:629-638.

du Lac S, Raymond JL, Sejnowski TJ, Lisberger SG (1995) Learning and memory in the vestibulo-ocular reflex. Annu Rev Neurosci 18:409-441.

Fernandez C, Goldberg J (1971) Physiology of peripheral neurons innervating semicircular canals of the squirrel monkey. II. Response to sinusoidal stimulation and dynamics of peripheral vestibular system. J Neurophysiol 34:661-675.

Highstein SM (1998) Role of the flocculus of the cerebellum in motor learning of the vestibulo-ocular reflex. Otolaryngol Head Neck Surg 119:212-220.

Ito M (1982) Cerebellar control of the vestibulo-ocular reflex - around the flocculus hypothesis. Annu Rev Neurosci 5:275-298.

Jahnsen H (1986) Electrophysiological characteristics of neurones in the guinea-pig deep cerebellar nuclei in vitro. J Physiol (Lond) 372:129-147.

Lisberger SG, Pavelko TA (1988) Brain stem neurons in modified pathways for motor learning in the primate vestibulo-ocular reflex. Science 242:771-773.

Lisberger SG, Pavelko TA, Broussard DM (1994a) Responses during eye movements of brain stem neurons that receive monosynaptic inhibition from the flocculus and ventral paraflocculus in monkeys. J Neurophysiol 72:909-927.

Lisberger SG, Pavelko TA, Broussard DM (1994b) Neural basis for motor learning in the vestibuloocular reflex of primates. I. Changes in the responses of brain stem neurons. J Neurophysiol 72:928-953.

Lisberger SG, Pavelko TA, Bronte-Stewart H, Stone LS (1994c) Neural basis for motor learning in the vestibulo-ocular reflex of primates: II. Changes in the responses of horizontal gaze velocity Purkinje cells in the cerebellar flocculus and ventral paraflocculus. J Neurophysiol 72:954-973.

Mauk MD (1997) Roles of cerebellar cortex and nuclei in motor learning: contradictions or clues? Neuron 18:343-346.

Mauk MD, Donegan NH (1997) A model of Pavlovian eyelid conditioning based on the synaptic organization of the cerebellum. Learn Mem 4:130-158.

Miles FA, Braitman DJ, Dow BM (1980) Long-term adaptive changes in primate vestibulo-ocular reflex. IV. Electrophysiological observations in flocculus of adapted monkeys. J Neurophysiol 43:1477-1493.

Mouginot D, Gahwiler BH (1995) Characterization of synaptic connections between cortex and deep nuclei of the rat cerebellum in vitro. Neuroscience 64:699-712.

Oberdick J, Smeyne RJ, Mann JR, Zackson S, Morgan JI (1990) A promoter that drives transgene expression in cerebellar Purkinje and retinal bipolar neurons. Science 248:223-226.

Palkovits M, Mezey E, Hamori J, Szentagothai J (1977) Quantitative histological analysis of the cerebellar nuclei in the cat. I. Numerical data on cells and on synapses. Exp Brain Res 28:189-209.

Partsalis AM, Zhang Y, Highstein SM (1995) Dorsal Y group in the squirrel monkey. I. Neuronal responses during rapid and long-term modifications of the vertical VOR. J Neurophysiol 73:615-631.

Raman IM, Gustafson AE, Padgett D (2000) Ionic currents and spontaneous firing in neurons isolated from the cerebellar nuclei. J Neurosci 20:9004-9016.

Raymond JL, Lisberger SG (1998) Neural learning rules for the vestibuloocular reflex. J Neurosci 18:9112-9129.

Raymond JL, Lisberger SG, Mauk MD (1996) The cerebellum: a neuronal learning machine? Science 272:1126-1131.

Sato Y, Kanda K, Kawasaki T (1988) Target neurons of floccular middle zone inhibition in medial vestibular nucleus. Brain Res 446:225-235.

Sekirnjak C, du Lac S (2002) Intrinsic firing dynamics of vestibular nucleus neurons. J Neurosci 22:2083-2095.

Telgkamp P, Raman IM (2002) Depression of inhibitory synaptic transmission between Purkinje cells and neurons of the cerebellar nuclei. J Neurosci 22:8447-8457.

Watanabe E (1984) Neuronal events correlated with long-term adaptation of the horizontal vestibulo-ocular reflex in the primate flocculus. Brain Res 297:169-174.

Williams RW, Rakic P (1988) Elimination of neurons from the rhesus monkey's lateral geniculate nucleus during development. J Comp Neurol 272:424-436.

Zhang Y, Partsalis AM, Highstein SM (1995) Properties of superior vestibular nucleus flocculus target neurons in the squirrel monkey. I. General properties in comparison with flocculus projecting neurons. J Neurophysiol 73:2261-2278. 